\title{
Discordance between radiographic findings, pain, and superficial temperature in knee osteoarthritis
}

\author{
Natália Cristina de Oliveira Vargas e Silva ${ }^{1}$, Rafael Luz dos Anjos ${ }^{1}$, \\ Matheus Miranda Campos Santana ${ }^{1}$, Linamara Rizzo Battistella ${ }^{2,3}$, Fábio Marcon Alfieri ${ }^{1,3}$ \\ ${ }^{1}$ Adventist University of São Paulo (UNASP), Brazil \\ ${ }^{2}$ School of Medicine, University of São Paulo, Brazil \\ ${ }^{3}$ Clinical Research Center, Institute of Physical Medicine and Rehabilitation, Clinics Hospital (HCFMUSP), School of Medicine, \\ University of São Paulo, Brazil
}

\begin{abstract}
Introduction: Knee pain is an imprecise marker of radiographic evidence of osteoarthritis (OA). These patients are more likely to develop central sensitisation to pain, a risk factor for chronic pain. Objectives: The aim of this study was to examine the associations among radiographic evidence of OA, self-reported pain, pressure pain thresholds (PPT), and superficial knee temperature in individuals with knee OA.

Material and methods: This cross-sectional study enrolled 25 patients with knee OA with clinic and radiographic diagnosis of bilateral OA (Kellgren-Lawrence grading scale 1-4 in X-ray images), but symptoms of pain and discomfort in only one of the knees, with pain perception in the symptomatic knee equal to or above 4 in a visual analogue scale. Volunteers underwent an evaluation including demographic data, superficial knee temperature (infrared thermography, mean superficial temperature of the region of the knee) and PPT (digital algometry at longus adductor, vastus lateralis, vastus medialis and tibialis anterior muscles, patellar tendon, and centre of the patella).

Results: Comparisons between symptomatic and asymptomatic knees revealed no differences regarding Kellgren-Lawrence classification, knee superficial temperature, or PPT. Significant weak and moderate associations were found between radiographic classification of OA and PPT of both knees in almost all sites evaluated. Nonetheless, superficial temperature of the knee was not associated with PPT or Kellgren-Lawrence grading scale.

Conclusions: Patients with bilateral knee OA presented no differences in symptomatic and asymptomatic knees regarding radiographic evidence, knee temperature, and PPT, indicating that central sensitisation may be present in them. Radiographic classification of OA was significantly associated with PPT in both knees.
\end{abstract}

Key words: osteoarthritis, pain threshold, thermography, rheumatology, knee.

\section{Introduction}

Osteoarthritis (OA) is the most common knee pathology in the elderly, and the most prevalent reason for knee pain and disability [1]. As a multifactorial disease, risk factors include genetics, female gender, obesity, and knee injury [2]. Although it is more prevalent among elderly people, nearly half the persons with symptomatic knee OA are between 45 and 64 years of age [3]. Early detection of this condition is important to prevent disability and reduce morbidity [4].

Standard assessment of joint degeneration is usually made by radiographic images that evaluate the presence of osteophytes and joint space narrowing [5]. However, individuals presenting knee pain do not always have radiographic evidence of $\mathrm{OA}$, and many asymptomatic patients may have severe structural damage in the knee joint [6].

Address for correspondence:

Fábio Marcon Alfieri, Clinical Research Center, Institute of Physical Medicine and Rehabilitation, University of São Paulo School of Medicine, Rua Domingo de Soto 100, Vila Mariana, 04116-030 São Paulo, Brazil, e-mail: fabioalfieri@usp.br; fabio.alfieri@unasp.edu.br Submitted: 16.06.2020; Accepted: 27.11.2020 
A systematic review of studies involving $X$-rays and clinical signs of OA [1] concluded that knee pain is an imprecise marker of radiographic evidence of OA; also, this radiographic evidence is an imprecise indicator of the likelihood that knee pain or disability will be present. This discrepancy may be explained by the susceptibility of some patients to develop central sensitisation to pain, a pain-amplifying neuroplastic alteration [5] considered to be a risk factor for developing chronic pain [7].

Conversely, a study involving two cohorts of the United States population has demonstrated a strong association between $\mathrm{X}$-ray findings and pain [8]. The authors evaluated self-reported pain using a specific instrument for patients with OA (WOMAC - Western Ontario and McMaster Universities Index).

Self-reported pain may be influenced by gender, ethnicity, age, and even culture [9]. Direct quantitative measures, such as pressure pain thresholds (PPT), are gold standard methods for evaluating pain [10]. A study involving quantitative sensory testing (pain thresholds) [5] observed that patients with elevated levels of clinical pain and no evidence of moderate to severe radiographic evidence of knee OA were the most pain sensitive, which suggests central sensitisation.

Pain in individuals with OA may also be due to an underlying inflammatory process responsible also for redness, heat, swelling, and joint stiffness [11]. Therefore, physiological and inflammation-related parameters are useful tools in the study of OA pain. Infrared thermography (IRT) is an accurate, noninvasive, and radiation-free technique to assess superficial temperature (ST) and indicate inflammation or changes in blood flow related to clinical abnormalities [12].

Superficial temperature in patients with OA has been little explored by the scientific literature so far. The study by Denoble et al. [13] found a moderate association between ST and pain, and Varjú et al. [14] verified a strong inverse association between superficial temperature and radiographic classification of $O A$.

Considering the controversial findings about X-ray severity and pain in knee OA, and the lack of studies exploring the associations between ST, pain, and radiographic findings, the aim of this study was to examine

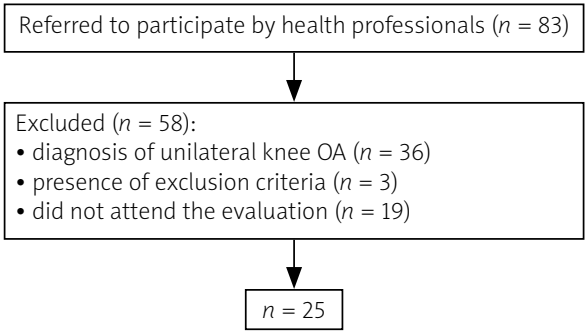

Fig. 1. Study flowchart. the associations among radiographic evidence of $O A$, self-reported pain, pressure pain thresholds, and superficial knee temperature in individuals with knee OA.

\section{Material and methods}

This was a cross-sectional study in which 83 patients of both genders with knee OA were invited to participate. Post hoc sample size estimate for an $\alpha=0.05$ resulted in a statistical power of $76 \%$.

Data collection was conducted at a University clinic from a private University. Patients referred to exercise treatment or physical therapy by the public primary health attention were invited to participate by the clinic's health professionals.

Inclusion criteria involved clinical and radiographic diagnosis of bilateral knee OA (Kellgren-Lawrence grading scale 1-4 in X-ray images in standard weight-bearing antero-posterior and lateral views of the knees in extension), symptoms of pain and discomfort in only one of the knees, and pain perception in the symptomatic knee equal to or above 4 in the VAS (visual analogue scale) [15]. Patients with any other chronic disease such as fibromyalgia, rheumatoid arthritis, neurologic or cardiac diseases, and uncontrolled hypertension were excluded from the study, as well as those with total or partial prosthesis in one or both knees or hips. Out of the 83 patients initially enrolled, 25 were eligible to participate in the study protocol (Fig. 1).

Volunteers underwent an evaluation including demographic data (age, gender, weight, and height), superficial knee temperature, and pressure pain tolerance thresholds (PPT).

Pressure pain tolerance thresholds were assessed by pressure algometry with a J Tech digital algometer (J Tech Medical, Salt Lake City, UT, USA). The device contains a rubber end of $1 \mathrm{~cm}^{2}$ in diameter. Pressure was applied at a constant rate of $1 \mathrm{~kg} / \mathrm{s}$ until pain or discomfort was reported by the volunteer. Participants were instructed to say "stop" as soon as the feeling of pressure changed from unpleasant to painful. The test was interrupted as soon as the volunteer indicated the onset of pain, and the final amount of force applied was recorded. For the evaluation, each participant remained in dorsal and lateral decubitus positions. Regions evaluated were as follows: longus adductor, vastus lateralis, vastus medialis and tibialis anterior muscles, patellar tendon, and centre of the patella. These sites have already been described and evaluated in previous studies $[16,17]$.

Superficial knee temperature was evaluated by thermography, in a room with controlled temperature and windows and curtains closed, blocking the entrance of external light, humidity, and air circulation. Environmental temperature variation was less than $1^{\circ} \mathrm{C}$ within 
a period of 20 minutes. The velocity of the incident air did not exceed $0.2 \mathrm{~m} / \mathrm{s}$. Relative air humidity was maintained at around $50 \%$. Because the conditions for this type of examination must be standardised, the study participants were instructed not to:

- take hot baths or showers, use creams or powder, or perform vigorous exercises in the 2 previous hours from data collection,

- take in stimulants, caffeinated or alcoholic beverages, make use of nasal decongestants, or to smoke,

- be fasting for more than two hours.

Once these requirements were met, volunteers were asked to wear shorts in order to expose their lower limbs. Prior to the collection of the thermographic image, each volunteer remained in the room for $15 \mathrm{~min}$ utes, to allow thermal equilibrium with the temperature of the examination room. The image was collected in the anterior incidence, with participants standing $2 \mathrm{~m}$ away from an infrared sensor (FLIR E4, Wilsonville, Oregon, USA). Images were analysed by the sensor manufacturer's software. The mean superficial temperature of the region of interest (ROI) of the knee (18) was obtained.

\section{Ethical standards}

This study was approved by local Ethics Committee (protocol number 1.815.849), and participants signed a written informed consent form.

\section{Statistical analyses}

Data analysis was conducted in SPSS v.24 for Windows. Normality of data was tested by the KolmogorovSmirnov method. Comparisons between symptomatic and asymptomatic knees were obtained by Wilcoxon or paired Student's t-tests. Associations between study variables were established by Spearman or Pearson correlation tests and classified as follows: 0.0 to 0.19 - very weak association; 0.2 to 0.39 - weak association; 0.4 to 0.69 - moderate association; 0.7 to 0.89 - strong association; and 0.9 to 1.0 - very strong association. In all cases, descriptive level $\alpha$ was set at 5\%.

\section{Results}

The study sample comprised 25 individuals with knee $\mathrm{OA}$, with ages ranging from 54 to 78 years. All of them presented radiographic evidence of bilateral knee OA, but symptoms of pain and discomfort were self-reported by patients in only one of the knees. Mean pain intensity measured by VAS was moderate (Table I).

Comparisons between symptomatic and asymptomatic knees revealed no differences regarding KellgrenLawrence radiographic classification, knee $\mathrm{ROI}$ superfi-
Table I. Demographic data

\begin{tabular}{|lc|}
\hline Age (years) & $66.0 \pm 5.2$ \\
\hline BMI & $31.1 \pm 3.0$ \\
\hline Gender (\% F) & $19(76.0)$ \\
\hline Symptomatic knee (\% R) & $9(36.0)$ \\
\hline VAS & $6.7 \pm 1.8$ \\
\hline
\end{tabular}

BMI - body mass index, F-female, VAS - visual analogue scale, $R$-right.

Data are presented as means \pm standard deviations or $n(\%)$.

Table II. Radiographic classification, pain, and temperature in symptomatic and asymptomatic knees

\begin{tabular}{|lccc|}
\hline Parameters & Symptomatic & Asymptomatic & $p$ \\
\hline Kellgren-Lawrence & $2.2 \pm 0.9$ & $2.1 \pm 0.7$ & $0.257^{*}$ \\
\hline $\begin{array}{l}\text { PPT adductor } \\
\text { longus (kgf) }\end{array}$ & $1.7 \pm 1.0$ & $1.7 \pm 0.9$ & $0.577^{*}$ \\
\hline $\begin{array}{l}\text { PPT vastus } \\
\text { lateralis (kgf) }\end{array}$ & $2.6 \pm 1.0$ & $2.9 \pm 1.3$ & $0.149^{*}$ \\
\hline $\begin{array}{l}\text { PPT vastus } \\
\text { medialis (kgf) }\end{array}$ & $2.3 \pm 1.3$ & $2.7 \pm 1.2$ & $0.128^{*}$ \\
\hline $\begin{array}{l}\text { PPT centre } \\
\text { of patella (kgf) }\end{array}$ & $2.7 \pm 1.2$ & $3.6 \pm 1.6$ & $0.006^{* *}$ \\
\hline $\begin{array}{l}\text { PPT patellar } \\
\text { tendon (kgf) }\end{array}$ & $3.5 \pm 2.4$ & $4.3 \pm 2.4$ & $0.016^{*}$ \\
\hline $\begin{array}{l}\text { PPT tibialis } \\
\text { anterior (kgf) }\end{array}$ & $3.2 \pm 1.5$ & $3.6 \pm 1.8$ & $0.092^{* *}$ \\
\hline $\begin{array}{l}\text { Knee } \\
\text { temperature }\left({ }^{\circ} \mathrm{C}\right)\end{array}$ & $31.2 \pm 1.4$ & $31.1 \pm 1.5$ & $0.379^{* *}$ \\
\hline PPT - pressurepan threshold. & & \\
\hline
\end{tabular}

PPT-pressure pain threshold.

Data are presented as means \pm standard deviations.

*Wilcoxon test, ${ }^{* *}$ paired Student's t-test.

cial temperature, or pain tolerance (except for the centre of the patella and patellar tendon), as shown in Table II.

However, significant weak and moderate associations were found between radiographic classification of OA and pain tolerance (of both knees) in almost all sites evaluated. Nonetheless, superficial temperature of the knee ROI was not associated with PPT or Kellgren-Lawrence grading scale (Table III).

\section{Discussion}

This study aimed at examining associations among radiographic evidence of OA, self-reported pain, PPT, and superficial temperature of the knee in individuals with knee OA. There were no differences between symptomatic and asymptomatic knees concerning KellgrenLawrence classification, knee temperature, or pain tolerance at most sites evaluated. Despite this finding, significant associations were found between radiographic 
Table III. Associations between study variables

\begin{tabular}{|c|c|c|}
\hline Parameters & $r$ & $p$ \\
\hline $\begin{array}{l}\text { Kellgren-Lawrence } \times \text { PPT } \\
\text { adductor longus (kgf) }\end{array}$ & -0.28 & $0.04^{*}$ \\
\hline $\begin{array}{l}\text { Kellgren-Lawrence } \times \text { PPT } \\
\text { vastus lateralis }(\mathrm{kgf})\end{array}$ & -0.21 & $0.14^{*}$ \\
\hline $\begin{array}{l}\text { Kellgren-Lawrence } \times \text { PPT } \\
\text { vastus medialis }(\mathrm{kgf})\end{array}$ & -0.28 & $0.05^{*}$ \\
\hline $\begin{array}{l}\text { Kellgren-Lawrence } \times \text { PPT } \\
\text { centre of patella }(\mathrm{kgf})\end{array}$ & -0.47 & $0.001^{*}$ \\
\hline $\begin{array}{l}\text { Kellgren-Lawrence } \times \text { PPT } \\
\text { patellar tendon }(\mathrm{kgf})\end{array}$ & -0.35 & $0.01^{*}$ \\
\hline $\begin{array}{l}\text { Kellgren-Lawrence } \times \text { PPT } \\
\text { tibialis anterior }(\mathrm{kgf})\end{array}$ & -0.46 & $0.001^{*}$ \\
\hline $\begin{array}{l}\text { Kellgren-Lawrence } \times \text { knee } \\
\text { temperature }\left({ }^{\circ} \mathrm{C}\right)\end{array}$ & 0.17 & $0.25^{*}$ \\
\hline Knee temperature $(\mathrm{OC}) \times$ VAS & 0.19 & $0.35^{*}$ \\
\hline $\begin{array}{l}\text { Knee temperature }(\mathrm{oC}) \times \mathrm{PPT} \\
\text { adductor longus }(\mathrm{kgf})\end{array}$ & -0.21 & $0.15^{\star}$ \\
\hline $\begin{array}{l}\text { Knee temperature }(\mathrm{oC}) \times \mathrm{PPT} \\
\text { vastus lateralis }(\mathrm{kgf})\end{array}$ & -0.12 & $0.41^{*}$ \\
\hline $\begin{array}{l}\text { Knee temperature }(\mathrm{oC}) \times \text { PPT } \\
\text { vastus medialis }(\mathrm{kgf})\end{array}$ & -0.23 & $0.12^{*}$ \\
\hline $\begin{array}{l}\text { Knee temperature }(\mathrm{oC}) \times \mathrm{PPT} \\
\text { center of patella }(\mathrm{kgf})\end{array}$ & -0.19 & $0.18^{\star *}$ \\
\hline $\begin{array}{l}\text { Knee temperature }(\mathrm{oC}) \times \mathrm{PPT} \\
\text { patellar tendon }(\mathrm{kgf})\end{array}$ & 0.06 & $0.67^{*}$ \\
\hline $\begin{array}{l}\text { Knee temperature }(\mathrm{oC}) \times \mathrm{PPT} \\
\text { tibialis anterior }(\mathrm{kgf})\end{array}$ & -0.24 & $0.09^{* *}$ \\
\hline Kellgren-Lawrence $\times$ VAS & 0.19 & $0.36^{*}$ \\
\hline Kellgren-Lawrence $\times \mathrm{BMI}$ & 0.02 & $0.91^{*}$ \\
\hline
\end{tabular}

classification of OA and pain tolerance of both knees, but knee temperature was not associated with PPT or radiographic evidence severity.

The mean BMI of patients was 31.1, classified as obesity. The association between obesity and prevalence of knee $O A$ is widely recognised, and body weight is the main modifiable risk factor for this disease [19]. However, the lack of association between BMI and radiographic evidence of OA observed in this study is not a novel finding [20].

Our data also confirmed the discordance between pain perception (VAS) and Kellgren-Lawrence classification, previously observed by several studies $[1,5,6]$. Thus, self-reported knee pain is an imprecise marker of radiographic knee OA [1]. The mean VAS of patients in the present study was 6.7 , which can be classified as moderate pain [15]. Mean Kellgren-Lawrence classification (on a scale of 1 to 4 ) of our patients was close to 2 , indicating possible narrowing of the joint space with definite osteophyte formation [21]. Notwithstanding the lack of association between $\mathrm{X}$-ray findings and self-reported pain, when analysing the above values, a mean moderate pain is probably in agreement with a mean Kellgren-Lawrence score of around 2.

In order to minimise the influence of subjective confounders on pain assessment, digital algometry, a gold standard method for evaluating pain, was conducted in this study. Symptomatic and asymptomatic knees were not different regarding Kellgren-Lawrence classification and PPT (at 4 out of the 6 sites evaluated). OA patients are prone to develop sensitised central nociceptive circuits that enhance pain during various states of peripheral tissue insult [5]. This may be shown in this study because patients tolerated the same pressure pain in the asymptomatic knee as in the painful limb.

Despite the discordance between VAS and Kellgren-Lawrence score, PPTs were found to be significantly associated with this latter variable. In contrast, previous studies found no significant associations between PPT and severity of radiographic OA $[22,23]$. Although PPT is a direct measure of pain, with an apparent advantage over self-reporting, pain might be influenced by several aspects [9] and thus deserve further investigations.

Out of the 6 points evaluated by algometry, only one (vastus lateralis muscle) did not correlate with radiographic evidence of OA. This was observed in the knee itself (centre of patella and patellar tendon) and in regions far from it (adductor longus, vastus medialis, and tibialis anterior muscles). Corroborating our data, a study that evaluated PPT in patients with knee and hip OA [22] observed that the presence of knee symptoms was associated with augmented pain processing (lower PPT), even at a distant site (upper trapezius muscle).

Pressure pain was not associated with the superficial temperature of the knee. Our data corroborates the pilot study by Tsai et al. [24], who also did not find significant associations between knee temperature and any measure of knee pain. However, surprisingly, superficial temperature of the knee ROI was also not associated with the Kellgren-Lawrence grading scale. Infrared images reflect thermal processes of the body [25], and it was expected that an altered joint temperature would be reflected in knee superficial temperature. This relationship has been previously demonstrated by Denoble et al. [3] and by Varjú et al. [14] in a study with patients with hand OA. The lack of association observed herein leads us to speculate that patients presented no active inflammatory mechanisms in the knee joint at the time of assessment, because superficial temperature evalu- 
ated by thermography may reflect increased synovitis or subchondral bone activity, or both [26].

This study has some limitations. The relatively small sample size may have limited the statistical power to detect effects, and the cross-sectional nature of the study prevents further cause-effect conclusions. Also, pain mechanisms in chronic diseases are complex and deserve thorough investigations. We did not evaluate changes in sensitisation of central structures, so we cannot unequivocally state that hyperalgesia was associated with brain changes due to chronic pain in our patients. Despite these limitations, our study provides important data on augmented central pain processing, possibly caused by chronic pain in the knee joint.

\section{Conclusions}

Patients with bilateral knee OA presented no differences in symptomatic and asymptomatic knees regarding Kellgren-Lawrence grading scale, knee temperature, and pain tolerance, indicating that central sensitisation may be present in the sample. Radiographic classification of OA was significantly associated with pain tolerance in both knees, but not with self-reported pain. Superficial knee temperature was not associated with pain or radiographic evidence severity.

Further studies should explore hyperalgesia in areas close to and distant from the affected joints. More studies with patients with bilateral OA but asymptomatic in one limb are desirable to increase the understanding of the underlying mechanisms of the nervous system that enhance patients' pain. These insights may encourage further studies on new therapeutic approaches to control pain in individuals with $O A$.

The authors declare no conflict of interests.

\section{References}

1. Bedson J, Croft PR. The discordance between clinical and radiographic knee osteoarthritis: a systematic search and summary of the literature. BMC Musculoskelet Disord 2008; 9: 116, DOI: 10.1186/1471-2474-9-116.

2. Silverwood V, Blagojevic-Bucknall M, Jinks C, et al. Current evidence on risk factors for knee osteoarthritis in older adults: a systematic review and meta-analysis. Osteoarthritis Cartilage 2015; 23: 507-515, DOI: 10.1016/j.joca.2014.11.019.

3. Deshpande BR, Katz JN, Solomon DH, et al. Number of persons with symptomatic knee osteoarthritis in the US: impact of race and ethnicity, age, sex, and obesity. Arthritis Care Res (Hoboken) 2016; 68: 1743-1750, DOI: 10.1002/acr.22897.

4. Lakkireddy M, Bedarakota D, Vidyasagar J, et al. Correlation among radiographic, arthroscopic and pain criteria for the diagnosis of knee osteoarthritis. J Clin Diagn Res 2015; 9: RC04RC07, DOI: 10.7860/JCDR/2015/17152.6889.
5. Finan PH, Buenaver LF, Bounds SC, et al. Discordance between pain and radiographic severity in knee osteoarthritis: findings from quantitative sensory testing of central sensitization. Arthritis Rheum 2013; 65: 363-372, DOI: 10.1002/art.34646.

6. Astephen Wilson JL, Stanish WD, Hubley-Kozey CL. Asymptomatic and symptomatic individuals with the same radiographic evidence of knee osteoarthritis walk with different knee moments and muscle activity. J Orthop Res 2017; 35: 1661-1670, DOI: 10.1002/jor.23465.

7. Nijs J, van Wilgen CP, Van Oosterwijck J, et al. How to explain central sensitization to patients with 'unexplained' chronic musculoskeletal pain: practice guidelines. Man Ther 2011; 16: 413-418, DOI: 10.1016/j.math.2011.04.005.

8. Neogi T, Felson D, Niu J, et al. Association between radiographic features of knee osteoarthritis and pain: results from two cohort studies. BMJ 2009; 339: b2844, DOI: 10.1136/bmj. b2844.

9. Torres CA, Bartley EJ, Wandner LD, et al. The influence of sex, race, and age on pain assessment and treatment decisions using virtual human technology: a cross-national comparison. J Pain Res 2013; 6: 577-588, DOI: 10.2147/JPR.S46295.

10. Egloff N, Klingler N, von Känel RV, et al. Algometry with a clothes peg compared to an electronic pressure algometer: a randomized cross-sectional study in pain patients. BMC Musculoskelet Disord 2011; 25: 174, DOI: 10.1186/1471-2474-12-174.

11. Dieppe P, Lohmander S. Pathogenesis and management of pain in osteoarthritis. Lancet 2005; 365: 965-973, DOI: 10.1016/ S0140-6736(05)71086-2.

12. Ludwig N, Formenti D, Gargano M, Alberti G. Skin temperature evaluation by infrared thermography: comparison of image analysis methods. Infrared Phys Technol 2013; 62: 1-6, DOI: 10.1016/j.infrared.2013.09.011.

13. Denoble AE, Hall N, Pieper CF, Kraus VB. Patellar skin surface temperature by thermography reflects knee osteoarthritis severity. Clin Med Insights Arthritis Musculoskelet Disord 2010; 3: 69-75, DOI: 10.4137/CMAMD.S5916.

14. Varjú G, Pieper CF, Renner JB, Kraus VB. Assessment of hand osteoarthritis: correlation between thermographic and radiographic methods. Rheumatology (Oxford) 2004; 43: 915-919, DOI: 10.1093/rheumatology/keh204.

15. Hawker GA, Mian S, Kendzerska T, French M. Measures of adult pain: Visual Analog Scale for Pain (VAS Pain), Numeric Rating Scale for Pain (NRS Pain), McGill Pain Questionnaire (MPQ), Short-Form McGill Pain Questionnaire (SF-MPQ), Chronic Pain Grade Scale (CPGS), Short Form-36 Bodily Pain Scale (SF-36 BPS), and Measure of Intermittent and Constant Osteoarthritis Pain (ICOAP). Arthritis Care Res (Hoboken) 2011; 63 Suppl 11: S240-S252, DOI: 10.1002/acr.20543.

16. Imamura M, Imamura ST, Kaziyama $\mathrm{HH}$, et al. Impact of nervous system hyperalgesia on pain, disability, and quality of life in patients with knee osteoarthritis: a controlled analysis. Arthritis Rheum 2008; 59: 1424-1431, DOI: 10.1002/art.24120.

17. Skou ST, Roos EM, Laursen MB, et al. A randomized, controlled trial of total knee replacement. N Engl J Med 2015; 373: 15971606, DOI: 10.1056/NEJMoa1505467.

18. Owen R, Ramlakhan S, Saatchi R, Burke D. Development of a high-resolution infrared thermographic imaging method as a diagnostic tool for acute undifferentiated limp in young chil- 
dren. Med Biol Eng Comput 2018; 56: 1115-1125, DOI: 10.1007/ s11517-017-1749-0.

19. Kulkarni K, Karssiens T, Kumar V, Pandit H. Obesity and osteoarthritis. Maturitas 2016; 89: 22-28, DOI: 10.1016/j.maturitas.2016.04.006.

20. Goulston LM, Kiran A, Javaid MK, et al. Does obesity predict knee pain over fourteen years in women, independently of radiographic changes? Arthritis Care Res (Hoboken) 2011; 63: 1398-1406, DOI: 10.1002/acr.20546.

21. Kohn MD, Sassoon AA, Fernando ND. Classifications in brief: Kellgren-Lawrence classification of osteoarthritis. Clin Orthop Relat Res 2016; 474: 1886-1893, DOI: 10.1007/s11999-016-4732-4.

22. Goode AP, Shi XA, Gracely RH, et al. Associations between pressure-pain threshold, symptoms, and radiographic knee and hip osteoarthritis. Arthritis Care Res (Hoboken) 2014; 66: 1513-1519, DOI: 10.1002/acr.22321.
23. Neogi T, Frey-Law L, Scholz J, et al., Multicenter Osteoarthritis (MOST) Study. Sensitivity and sensitisation in relation to pain severity in knee osteoarthritis: trait or state? Ann Rheum Dis 2015; 74: 682-688, DOI: 10.1136/annrheumdis-2013-204191.

24. Tsai PF, Richards K, Tatom I. The association between knee temperature and pain in elders with osteoarthritis of the knee: a pilot study. J Adv Nurs 2003; 42: 373-381, DOI: 10.1046/j.13652648.2003.02629.x

25. Vainer BG. FPA-based infrared thermography as applied to the study of cutaneous perspiration and stimulated vascular response in humans. Phys Med Biol 2005; 50: R63-R94, DOI: 10.1088/0031-9155/50/23/R01.

26. Warashina H, Hasegawa Y, Tsuchiya H, et al. Clinical, radiographic, and thermographic assessment of osteoarthritis in the knee joints. Ann Rheum Dis 2002; 61: 852-854, DOI: 10.1136/ard.61.9.852. 